\title{
WARING'S PROBLEM FOR POLYNOMIALS IN TWO VARIABLES
}

\author{
ARNAUD BODIN AND MIREILLE CAR
}

(Communicated by Matthew A. Papanikolas)

\begin{abstract}
We prove that all polynomials in several variables can be decomposed as the sums of $k$ th powers: $P\left(x_{1}, \ldots, x_{n}\right)=Q_{1}\left(x_{1}, \ldots, x_{n}\right)^{k}+\cdots+$ $Q_{s}\left(x_{1}, \ldots, x_{n}\right)^{k}$, provided that elements of the base field are themselves sums of $k$ th powers. We also give bounds for the number of terms $s$ and the degree of the $Q_{i}^{k}$. We then improve these bounds in the case of two-variable polynomials of large degree to get a decomposition $P(x, y)=Q_{1}(x, y)^{k}+\cdots+Q_{s}(x, y)^{k}$ with $\operatorname{deg} Q_{i}^{k} \leqslant \operatorname{deg} P+k^{3}$ and $s$ that depends on $k$ and $\ln (\operatorname{deg} P)$.
\end{abstract}

\section{INTRODUCTION}

For any domain $A$ and any integer $k \geqslant 2$, let $W(A, k)$ denote the subset of $A$ formed by all finite sums of $k$ th powers $a^{k}$ with $a \in A$. Let $\underline{w}_{A}(k)$ denote the least integer $s$, if it exists, such that for every element $a \in W(A, k)$, the equation

$$
a=a_{1}^{k}+\cdots+a_{s}^{k}
$$

admits solutions $\left(a_{1}, \ldots, a_{s}\right) \in A^{s}$.

The case of polynomial rings $K[t]$ over a field $K$ is of particular interest (see [11, 7]). The similarity between the arithmetic of the ring $\mathbb{Z}$ and the arithmetic of the polynomial rings in a single variable $F[t]$ over a finite field $F$ with $q$ elements led us to investigate a restricted variant of Waring's problem over $F[t]$, namely the strict Waring problem. For $P \in F[t]$, a representation

$$
P=Q_{1}^{k}+\cdots+Q_{s}^{k} \quad \text { with } \operatorname{deg} Q_{i}^{k}<\operatorname{deg} P+k,
$$

and $Q_{i} \in F[t]$ is a strict representation.

For the strict Waring problem, analogs to the classical numbers $g_{\mathbb{N}}(k)$ and $G_{\mathbb{N}}(k)$ have been defined as follows. Let $g_{F[t]}(k)$ (resp. $\left.G_{F[t]}(k)\right)$ denote the least integer $s$, if it exists, such that every polynomial in $W(F[t], k)$ (resp. every polynomial in $W(F[t], k)$ of sufficiently large degree) may be written as a sum satisfying the strict degree condition.

Received by the editors June 16, 2011 and, in revised form, September 7, 2011.

2010 Mathematics Subject Classification. Primary 11P05; Secondary 13B25, 11T55.

Key words and phrases. Several variables polynomials, sum of powers, approximate roots, Vandermonde determinant. 
General results about Waring's problem for the ring of polynomials over a finite field may be found in [10, [1], [12, [13, 9] for the unrestricted problem and in [14, 8], 5], 3], 7] for the strict Waring problem. Gallardo's method introduced in [6] and performed in 4] to deal with Waring's problem for cubes was generalized in [3] and [7, where bounds for $g_{F[t]}(k)$ and $G_{F[t]}(k)$ were established when $q$ and $k$ satisfy some conditions.

The goal of this paper is a study of Waring's problem for the ring $F[x, y]$ of polynomials in two variables over a field $F$. As for the one-variable case, two variations of Waring's problem may be considered. The first one is the unrestricted Waring's problem; the second one takes degree conditions into account.

In Section 2 we start by some relations between Waring's problem for polynomials in one variable and Waring's problem for polynomials in $n \geqslant 2$ variables. In Section 3, we prove that, provided all elements of the field $F$ are sums of $k$ th powers, there exists a positive integer $s$ (depending on $F$ and $k$ ) such that every polynomial $P \in F[x, y]$ may be written as a sum

$$
P=Q_{1}^{k}+\cdots+Q_{s}^{k}
$$

where for $i=1, \ldots, s, Q_{i}$ is a polynomial of $K[x, y]$ such that $\operatorname{deg} Q_{i} \leqslant \operatorname{deg} P$. We then prove various improvements, the goal being to have in representations (†) a decomposition with the following properties: the first priority is to have the lowest possible degree for the polynomials $Q_{i}$ and the second priority is a small number of terms. In Section 5, we prove that (†) is possible for polynomials of large degree with $\operatorname{deg} Q_{i}^{k} \leqslant \operatorname{deg} P+k^{3}$, the number $s$ of terms depending on $F, k$ and $\operatorname{deg} P$. To do that, in Section 4 , we introduce the notion of an approximate root.

Let $F$ be a field such that: $F$ has more than $k$ elements, the characteristic of $F$ does not divide $k$ and each element of $F$ can be written as a sum of $w_{F}(k) k$ th powers of elements of $F$. We summarize in Table 1 the different bounds we get for a decomposition of a polynomial $P(x, y)$ of degree $d$ as a sum $P=\sum_{i=1}^{s} Q_{i}^{k}$.

TABLE 1

\begin{tabular}{|c|c|c|}
\cline { 2 - 3 } \multicolumn{1}{c|}{} & $\operatorname{deg} Q_{i}^{k}$ & $s$ \\
\hline Corollary 4 & $k d$ & $k w_{F}(k)$ \\
\hline Proposition [5 & $d+2(k-1)^{2}$ & $\frac{1}{2} k(d+1)(d+2) w_{F}(k)$ \\
\hline Proposition 6 & $2 d+4 k^{2}$ & $k^{2}(2 k-1) w_{F}(k)$ \\
\hline Theorem 8 & $d+k^{3}$ & $2 k^{3} \ln \left(\frac{d}{k}+1\right) \ln (2 k)+7 k^{4} \ln (k) w_{F}(k)^{2}$ \\
\hline
\end{tabular}

The two basic results are Corollary 4 (which gives a decomposition with very few terms of high degree) and Proposition 5 with many terms of low degree. Our first main result is Proposition 6, which provides a decomposition with terms of medium degree, but the number of terms depends only on $k$ and not on the degree of $P$. Then Theorem 8 decomposes $P$, of sufficiently large degree $d \geqslant 2 k^{4}$, into a sum of few terms of low degree.

For instance, given a field with $w_{F}(k)=1$ (that is to say, each element of $F$ is a $k$ th power $)$, set $d=200$ and $k=3$. Then each polynomial $P(x, y)$ of degree 200 can be written as $P=\sum_{i=1}^{s} Q_{i}^{3}$ with the values given in Table 21

\footnotetext{
${ }^{1}$ In fact the last bound comes from a sharper bound obtained in the proof of Theorem 8
} 
TABLE 2

\begin{tabular}{|c|c|c|}
\cline { 2 - 3 } \multicolumn{1}{c|}{} & $\operatorname{deg} Q_{i}^{k}$ & $s$ \\
\hline Corollary 4 & 600 & 3 \\
\hline Proposition 5 & 208 & 60903 \\
\hline Proposition 6 & 436 & 45 \\
\hline Theorem 8 & 227 & 812 \\
\hline
\end{tabular}

\section{The unRestricted WARING'S PROBlem}

If $A$ is a domain, we denote by $W(A, k, s)$ the set of elements $a \in A$ that can be written as a sum $a=a_{1}^{k}+\cdots+a_{s}^{k}$ with $a_{1}, \ldots, a_{s} \in A$; if $A=W(A, k, s)$ for an integer $s$, then for any integer $s^{\prime} \geqslant s$, we have $A=W\left(A, k, s^{\prime}\right)$. Let $w_{A}(k)$ denote the least integer $s$ such that $A=W(A, k, s)$. If such an $s$ does not exist, let $w_{A}(k)=\infty$. Observe that $w_{A}(k) \geqslant \underline{w}_{A}(k)$ and in the case that $A=W(A, k)$, then $w_{A}(k)=\underline{w}_{A}(k)$. In this section we are concerned with rings of polynomials in $n \geqslant 1$ variables.

Lemma 1. Let $A$ be a domain and let $s$ be a positive integer.

(1) If $A[t]=W(A[t], k, s)$, then $A=W(A, k, s)$, so that $w_{A}(k) \leqslant w_{A[t]}(k)$.

(2) $A[t]=W(A[t], k, s)$ if and only if $A\left[x_{1}, \ldots, x_{n}\right]=W\left(A\left[x_{1}, \ldots, x_{n}\right], k, s\right)$, so that $w_{A\left[x_{1}, \ldots, x_{n}\right]}(k)=w_{A[t]}(k)$.

A kind of reciprocal to (1) will be discussed later in Proposition 3.

Proof.

(1) Suppose $A[t]=W(A[t], k, s)$. Every $a \in A$ is a sum $a=Q_{1}^{k}+\cdots+Q_{s}^{k}$ for some $Q_{i} \in A[t]$. Specializing $t$ at 1 , for instance, gives $a=Q_{1}(1)^{k}+\cdots+$ $Q_{s}(1)^{k}$, a sum in $A$. Therefore, $w_{A[t]}(k) \geqslant w_{A}(k)$.

(2) (a) If $A[t]=W(A[t], k, s)$, then there exist $Q_{1}, \ldots, Q_{s} \in A[t]$ such that $t=Q_{1}(t)^{k}+\cdots+Q_{s}(t)^{k}$. Pick $P \in A\left[x_{1}, \ldots, x_{n}\right]$ and substitute $P$ for $t$. We get $P\left(x_{1}, \ldots, x_{n}\right)=Q_{1}\left(P\left(x_{1}, \ldots, x_{n}\right)\right)^{k}+\cdots+Q_{s}\left(P\left(x_{1}, \ldots, x_{n}\right)\right)^{k}$. Hence $w_{A\left[x_{1}, \ldots, x_{n}\right]}(k) \leqslant w_{A[t]}(k)$.

(b) If $A\left[x_{1}, \ldots, x_{n}\right]=W\left(A\left[x_{1}, \ldots, x_{n}\right], k, s\right)$, then any $P(t) \in A[t]$ can be written $P(t)=Q_{1}\left(t, x_{2}, \ldots, x_{n}\right)^{k}+\cdots+Q_{s}\left(t, x_{2}, \ldots, x_{n}\right)^{k}$. By the specialization $x_{2}=\cdots=x_{n}=1$ we get that $P(t) \in W(A[t], k, s)$. Therefore $w_{A\left[x_{1}, \ldots, x_{n}\right]}(k) \geqslant w_{A[t]}(k)$.

Remark. It is also true that $A[t]=W(A[t], k, s)$ if and only if $t \in W(A[t], k, s)$.

This remark motivates the fact that we consider Waring's problem for a polynomial ring $F\left[x_{1}, \ldots, x_{n}\right]$ where $F$ is a field satisfying the condition $F=W(F, k)$. Such a field is called a Waring field for the exponent $k$ or, briefly, a $k$-Waring field.

Let us give some examples. An algebraically closed field $F$ is a $k$-Waring field with $w_{F}(k)=1$ for every positive integer $k$. If $F$ is a finite field of characteristic $p$, for every positive integer $n, F$ is a $p^{n}$-Waring field with $w_{F}\left(p^{n}\right)=1$. It is known (cf. [1, [5]) that for a finite field $F$ of characteristic $p$ that does not divide $k$ and order $q=p^{m}, F$ is a Waring field for the exponent $k$ if and only if for all $d \neq m$ dividing $m,(q-1) /\left(p^{d}-1\right)$ does not divide $k$. 
When $F$ has prime characteristic $p$, it is sufficient to consider Waring's problem for exponents $k$ coprime with $p$. Indeed, we have

Proposition 2. Let $k \geqslant 2$ be coprime with $p$. Then, for any positive integer $\nu$ and for any positive integer $s$, we have

$$
\begin{gathered}
W\left(F\left[x_{1}, \ldots, x_{n}\right], k p^{\nu}, s\right)=\left\{Q^{p^{\nu}} \mid Q \in W\left(F\left[x_{1}, \ldots, x_{n}\right], k, s\right)\right\}, \\
w_{F\left[x_{1}, \ldots, x_{n}\right]}\left(k p^{\nu}\right)=w_{F\left[x_{1}, \ldots, x_{n}\right]}(k) .
\end{gathered}
$$

The proof is similar to that of [3, Theorem 2.1] and relies on the relation $\left(Q_{1}^{k}+\right.$ $\left.\cdots+Q_{s}^{k}\right)^{p}=Q_{1}^{p k}+\cdots+Q_{s}^{p k}$.

\section{VANDERMONDE DETERMINANTS}

3.1. Sum with high degree. Let us recall that for $\left(\alpha_{1}, \ldots, \alpha_{n}\right) \in L^{n}$, where $L$ is a field containing $F$, Vandermonde's determinant $V\left(\alpha_{1}, \ldots, \alpha_{n}\right)$ is

$$
V\left(\alpha_{1}, \ldots, \alpha_{n}\right):=\left|\begin{array}{ccccc}
1 & \alpha_{1} & \alpha_{1}^{2} & \cdots & \alpha_{1}^{n-1} \\
1 & \alpha_{2} & \alpha_{2}^{2} & \cdots & \alpha_{2}^{n-1} \\
\vdots & & & & \vdots \\
1 & \alpha_{n} & \alpha_{n}^{2} & \cdots & \alpha_{n}^{n-1}
\end{array}\right|=\prod_{1 \leqslant i<j \leqslant n}\left(\alpha_{i}-\alpha_{j}\right) .
$$

Proposition 3. Let $F$ be a field with more than $k$ elements, whose characteristic does not divide $k$, such that each element of $F$ can be written as a sum of $k$ th powers of elements of $F$. Then any polynomial $P\left(x_{1}, \ldots, x_{n}\right)$ with coefficients in the field $F$ is a sum of $k$ th powers. In other words, for any positive integer $n$,

$$
F\left[x_{1}, \ldots, x_{n}\right]=W\left(F\left[x_{1}, \ldots, x_{n}\right], k\right) .
$$

Proof. The proof follows ideas from [7]. Let $\alpha_{1}, \ldots, \alpha_{k}$ be distinct elements of $F$. First notice that by formula (1), if $t$ is any transcendental element over $F$, $V\left(\alpha_{1}, \ldots, \alpha_{k}\right)=V\left(t+\alpha_{1}, \ldots, t+\alpha_{k}\right)$. By expanding the determinant $V(t+$ $\alpha_{1}, \ldots, t+\alpha_{k}$ ) along the last column we get (a term marked $\check{x}_{i}$ means that it is omitted):

$$
\begin{aligned}
V\left(\alpha_{1}, \ldots, \alpha_{k}\right) & =V\left(t+\alpha_{1}, \ldots, t+\alpha_{k}\right) \\
& = \pm \sum_{i=1}^{k}(-1)^{i}\left(t+\alpha_{i}\right)^{k-1} V(t+\alpha_{1}, \ldots, \overbrace{t+\alpha_{i}}^{\vee}, \ldots, t+\alpha_{k}) \\
& = \pm \sum_{i=1}^{k}(-1)^{i}\left(t+\alpha_{i}\right)^{k-1} V\left(\alpha_{1}, \ldots, \check{\alpha}_{i}, \ldots, \alpha_{k}\right) .
\end{aligned}
$$

The constant $\gamma=V\left(\alpha_{1}, \ldots, \alpha_{k}\right)$ is non-zero since the $\alpha_{i}$ are distinct elements of $F$. We write

$$
\sum_{i=1}^{k} \frac{\left(t+\alpha_{i}\right)^{k-1}}{\beta_{i}}=\gamma,
$$

where the $\beta_{i}$ are non-zero constants in $F$. This formula proves that the function $C(t)=\sum_{i=1}^{k} \frac{\left(t+\alpha_{i}\right)^{k}}{\beta_{i}}-\gamma k t$ has an identically null derivative; since the characteristic 
of $F$ does not divide $k$, this implies that $C(t)$ is a constant, so that, for some $\delta \in F$,

$$
\sum_{i=1}^{k} \frac{\left(t+\alpha_{i}\right)^{k}}{\beta_{i}}=\gamma k t+\delta .
$$

Let $P\left(x_{1}, \ldots, x_{n}\right) \in F\left[x_{1}, \ldots, x_{n}\right]$. By substitution of $t$ by $(P-\delta) /(\gamma k)$ in equality (2) we get $P=\sum_{i=1}^{k} \frac{\left(P-\delta+\alpha_{i} \gamma k\right)^{k}}{\beta_{i}(\gamma k)^{k}}$. But by assumption $1 / \beta_{i}(\gamma k)^{k}$ is a sum of $k$ th powers of elements of $F$, so that $P\left(x_{1}, \ldots, x_{n}\right)$ is also a sum of $k$ th powers of elements of $F\left[x_{1}, \ldots, x_{n}\right]$.

Corollary 4. Let $F$ have more than $k$ distinct elements such that its characteristic does not divide $k$. Every polynomial $P\left(x_{1}, \ldots, x_{n}\right) \in F\left[x_{1}, \ldots, x_{n}\right]$ of degree $d$ can be written as a sum

$$
P\left(x_{1}, \ldots, x_{n}\right)=\delta_{1} Q_{1}\left(x_{1}, \ldots, x_{n}\right)^{k}+\cdots+\delta_{k} Q_{k}\left(x_{1}, \ldots, x_{n}\right)^{k},
$$

where $\delta_{1}, \ldots, \delta_{k} \in F$ and $Q_{1}, \ldots, Q_{k}$ are polynomials in $F\left[x_{1}, \ldots, x_{n}\right]$ such that $\operatorname{deg} Q_{i}^{k} \leqslant k d$. If moreover each element of $F$ is a sum of $w_{F}(k) k$ th powers, then

$$
P\left(x_{1}, \ldots, x_{n}\right)=Q_{1}\left(x_{1}, \ldots, x_{n}\right)^{k}+\cdots+Q_{s}\left(x_{1}, \ldots, x_{n}\right)^{k},
$$

where $Q_{1}, \ldots, Q_{s} \in F\left[x_{1}, \ldots, x_{n}\right]$ such that $\operatorname{deg}{Q_{i}}^{k} \leqslant k d$ for some $s \leqslant k \cdot w_{F}(k)$.

Proof. The proof comes from formula (2) and the discussion below it.

In the sequel, we consider polynomials in two variables.

\subsection{Low degree, many terms.}

Proposition 5. Let $F$ be a field with more than $k$ distinct elements such that its characteristic does not divide $k$. Every polynomial $P \in F[x, y]$ of degree $d$ admits a decomposition:

$$
P(x, y)=\delta_{1} Q_{1}(x, y)^{k}+\cdots+\delta_{s} Q_{s}(x, y)^{k},
$$

where $\delta_{1}, \ldots, \delta_{s} \in F$ and $Q_{1}, \ldots, Q_{s}$ are polynomials in $F[x, y]$ such that $\operatorname{deg} Q_{i}^{k} \leqslant$ $d+2(k-1)^{2}$ and $s \leqslant k \cdot \frac{(d+1)(d+2)}{2}$.

If moreover each element of $F$ is a sum of $k$ th powers, then $P$ admits a decomposition:

$$
P(x, y)=Q_{1}(x, y)^{k}+\cdots+Q_{s}(x, y)^{k},
$$

where $Q_{1}, \ldots, Q_{s} \in F[x, y]$ with $\operatorname{deg} Q_{i}^{k} \leqslant d+2(k-1)^{2}$ and $s \leqslant k w_{F}(k) \frac{(d+1)(d+2)}{2}$.

Proof. Let $P(x, y)=\sum a_{i, j} x^{i} y^{j}$. We make the Euclidean divisions: $i=p k+a$ and $j=q k+b$ with $0 \leqslant a, b<k$. Each monomial $x^{i} y^{j}$ can now be written $x^{i} y^{j}=\left(x^{p} y^{q}\right)^{k} \cdot x^{a} y^{b}$. By Corollary 4, $x^{a} y^{b}$ can be written $x^{a} y^{b}=\delta_{1} Q_{1}(x, y)^{k}+$ $\cdots+\delta_{k} Q_{k}(x, y)^{k}$ with $\delta_{1}, \ldots, \delta_{k} \in F, Q_{1}, \ldots, Q_{k} \in F[x, y]$ and $\operatorname{deg} Q_{i} \leqslant \operatorname{deg}\left(x^{a} y^{b}\right)$, so that

$$
x^{i} y^{j}=\delta_{1}\left(x^{p} y^{q} Q_{1}(x, y)\right)^{k}+\cdots+\delta_{k}\left(x^{p} y^{q} Q_{1}(x, y)\right)^{k} .
$$

Moreover $\operatorname{deg}\left(\left(x^{p} y^{q} Q_{i}(x, y)\right)^{k}\right)=k\left(p+q+\operatorname{deg} Q_{i}\right) \leqslant k p+k q+k a+k b=i+j+$ $(k-1)(a+b) \leqslant i+j+2(k-1)^{2} \leqslant d+2(k-1)^{2}$.

As $\operatorname{deg} P=d$ the number of monomials $x^{i} y^{j}$ is less than or equal to $\frac{(d+1)(d+2)}{2}$, so that $P$ admits a decomposition $P(x, y)=\delta_{1} Q_{1}(x, y)^{k}+\cdots+\delta_{s} Q_{s}(x, y)^{k}$ with $\operatorname{deg} Q_{i}^{k} \leqslant d+2(k-1)^{2}$ and $s \leqslant k \frac{(d+1)(d+2)}{2}$. Thus we can find a decomposition $P(x, y)=Q_{1}(x, y)^{k}+\cdots+Q_{s}(x, y)^{k}$ for some $s \leqslant k w_{F}(k) \frac{(d+1)(d+2)}{2}$. 
3.3. Medium degree, few terms. We improve this method to get fewer terms in the sum, but the degree of each term is higher.

Proposition 6. Let $F$ be a field with more than $k$ elements such that its characteristic does not divide $k$ and each element of $F$ is a sum of $k$ th powers. For any $P \in F[x, y], P$ admits a decomposition:

$$
P(x, y)=Q_{1}(x, y)^{k}+\cdots+Q_{s}(x, y)^{k},
$$

where $Q_{1}, \ldots, Q_{s}$ are polynomials in $F[x, y]$ with $\operatorname{deg} Q_{i}^{k} \leqslant 2 \operatorname{deg} P+4 k^{2}$ and $s \leqslant$ $k^{2}(2 k-1) w_{F}(k)$.

Observe that the bound for $s$ does not depend on the degree of the polynomial $P$.

Proof. Let $d$ be the least multiple of $2 k^{2}$ such that $d \geqslant \operatorname{deg} P$. The Newton polygon of $P$ is included in the triangle $A B C$ with $A(0,0), B(0, d), C(d, 0)$.

We cover this triangle $A B C$ by $k(2 k-1)$ small triangles that are translations (by $\frac{d}{2 k}$-units) of $A^{\prime} B^{\prime} C^{\prime}$ with $A^{\prime}(0,0), B^{\prime}\left(0, \frac{d}{k}\right), C^{\prime}\left(\frac{d}{k}, 0\right)$. See Figure 1. This covering

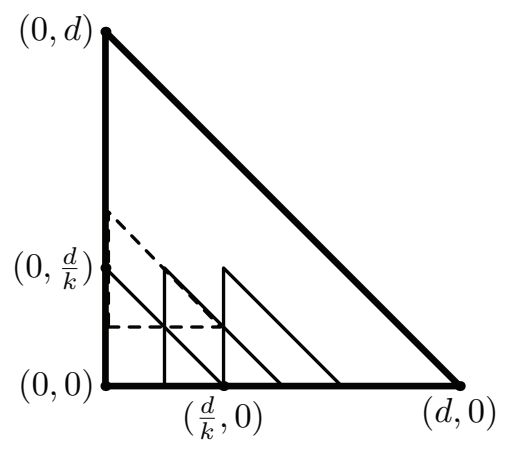

FIGURE 1

means that we can write $P(x, y)$ as a sum of $k(2 k-1)$ polynomials of the form $x^{i \frac{d}{2 k}} y^{j \frac{d}{2 k}} P_{i, j}(x, y)$ with $\operatorname{deg} P_{i, j} \leqslant \frac{d}{k}$ and $0 \leqslant i+j \leqslant 2 k-2$ (so that $\operatorname{deg} x^{i \frac{d}{2 k}} y^{j \frac{d}{2 k}}<$ d). As $2 k^{2}$ divides $d$, then $x^{i \frac{d}{2 k}} y^{j \frac{d}{2 k}}$ is a $k$ th power. Furthermore, by Corollary 4 , we can write each $P_{i, j}$ as a sum of $k w_{F}(k)$ powers, each power being of degree at most $k \frac{d}{k}=d$. Hence we get a decomposition $P(x, y)=Q_{1}(x, y)^{k}+\cdots+Q_{s}(x, y)^{k}$ with $s \leqslant k^{2}(2 k-1) w_{F}(k)$ terms and $\operatorname{deg} Q_{i}^{k}<2 d$.

\section{Approximate root}

In this section $F$ is a field whose characteristic does not divide $k$. Let $P \in F[x, y]$ be a polynomial that satisfies the following conditions: $\operatorname{deg} P \leqslant d, \operatorname{deg}_{x} P<m$, so that the Newton polygon $\Gamma(P)$ of $P$ is (included in) the following polygon $\bar{\Gamma}(P)$ (whose vertices are $(0,0),(m, 0),(m, n),(0, d))$. See Figure 2, We set $n=d-m$ and we suppose that $k|m, k| n, k \mid d$. We will look for a $Q \in F[x, y]$ such that $\operatorname{deg} Q \leqslant \frac{d}{k}, \operatorname{deg}_{x} Q \leqslant \frac{m}{k}$, so that $\Gamma\left(Q^{k}\right) \subset \bar{\Gamma}(P)$. In fact the Newton polygon of $Q$ is homothetic to the one of $P$ with a ratio $\frac{1}{k}$. 


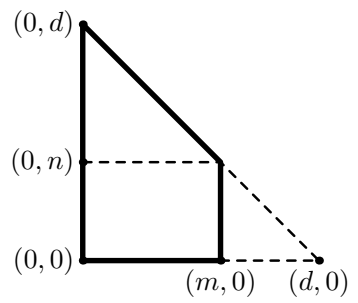

FiguRE 2

Proposition 7. There exists a unique $Q(x, y) \in F[x, y]$, monic in $x$, such that $P+x^{m} y^{n}-Q^{k}$ has no monomial $x^{i} y^{j}$ with $i \geqslant m-\frac{m}{k}$ and $j \geqslant n-\frac{n}{k}$. That is to say, the Newton polygon of $P+x^{m} y^{n}-Q^{k}$ is (included in) Figure 3 .

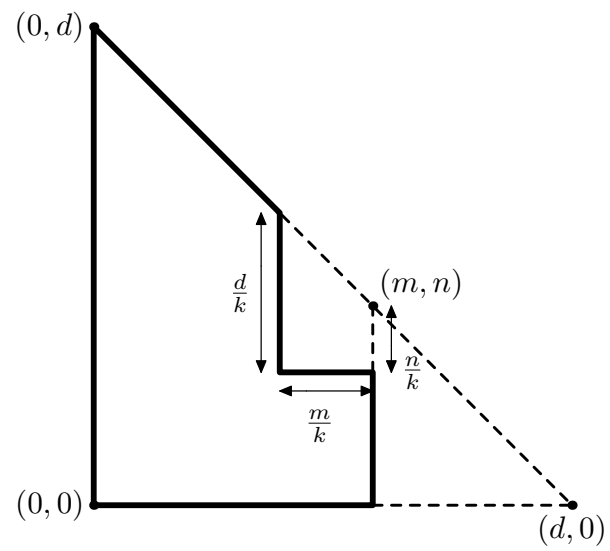

Figure 3

This means that with two $k$ th powers $\left(x^{m} y^{n}\right.$ and $\left.Q^{k}\right)$ we "cancel" the trapezium $T$ (defined by the vertices $\left.(m, n),\left(m, n-\frac{n}{k}\right),\left(m-\frac{m}{k}, n-\frac{n}{k}\right),\left(m-\frac{m}{k}, n+\frac{d}{k}-\frac{n}{k}\right)\right)$. This procedure is similar to the computation of the approximate $k$ th root of a one variable polynomial; see 2. The proof is sketched in Figure 4 .

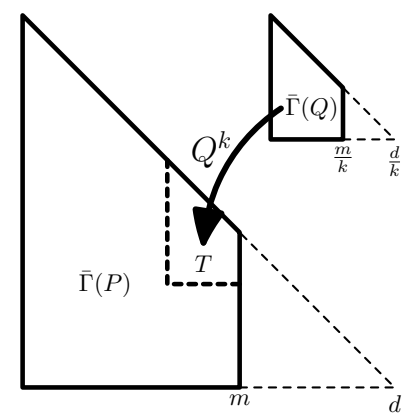

FiguRE 4 
Morally, the coefficients of $Q$ provide a set of unknowns, which are chosen in order that $Q^{k}$ and $P$ can be identified in the trapezium area $(T)$.

Proof. We write $P$ as the sum $P=P_{1}+P_{2}$ corresponding to the decomposition into two areas of $\bar{\Gamma}(P)=T \cup(\bar{\Gamma}(P) \backslash T)$ : we write $P_{1}$ as a polynomial in $x$ whose coefficients are in $F[y]$ so that $P_{1}(x, y)=a_{1}(y) x^{m-1}+\cdots+a_{\frac{m}{k}}(y) x^{m-\frac{m}{k}}$ with $\operatorname{deg} a_{i}(y) \leqslant n+i$ and val $a_{i}(y) \geqslant n-\frac{n}{k}$. We denote by val the $y$-adic valuation $\operatorname{val} \sum \alpha_{i} y^{i}=\min \left\{i \mid \alpha_{i} \neq 0\right\}$.

We set $P_{1}^{\prime}(x, y)=y^{n} x^{m}+P_{1}(x, y)$ and $a_{0}(y)=y^{n}$. Notice that we have added a $k$ th power since $k \mid m$ and $k \mid n$.

We also write $Q(x, y)$ as a polynomial in $x$ with coefficients in $F[y]: Q(x, y)=$ $b_{0}(y) x^{\frac{m}{k}}+b_{1}(y) x^{\frac{m}{k}-1}+\cdots+b_{\frac{m}{k}}(y)$.

We now identify the monomials of $P_{1}^{\prime}(x, y)=x^{m} y^{n}+P_{1}(x, y)$ with the monomials of $Q(x, y)^{k}$ in the trapezium $T$. As we only want to identify the monomials of a sufficiently high degree, we define the following equivalence:

$$
a(y) \bumpeq b(y) \quad \text { if and only if } \operatorname{deg}(a(y)-b(y))<n-\frac{n}{k} .
$$

This yields the following polynomial system of equations $\left(a_{i}(y)\right.$ are data, and $b_{i}(y)$ unknowns):

$$
\left\{\begin{array}{l}
a_{0} \bumpeq b_{0}^{k} \\
a_{1} \bumpeq k b_{0}^{k-1} b_{1} \\
a_{2} \bumpeq k b_{0}^{k-1} b_{2}+\left(\begin{array}{c}
k \\
2
\end{array}\right) b_{0}^{k-2} b_{1}^{2} \\
\vdots \\
a_{\ell} \bumpeq k b_{0}^{k-1} b_{\ell}+\sum_{\substack{i_{1}+2 i_{2}+\cdots+(\ell-1) i_{\ell-1}=\ell \\
i_{0}+i_{1}+i_{2}+\cdots+i_{\ell-1}=k}} c_{i_{1} \ldots i_{\ell-1}} b_{0}^{i_{0}} b_{1}^{i_{1}} \cdots b_{\ell-1}^{i_{\ell-1}}, \quad 1 \leqslant \ell \leqslant \frac{m}{k},
\end{array}\right.
$$

where the coefficients $c_{i_{1} \ldots i_{\ell-1}}$ are the multinomial coefficients defined by the following formula:

$$
c_{i_{1} \ldots i_{\ell-1}}=\left(\begin{array}{c}
k \\
i_{1}, \ldots, i_{\ell-1}
\end{array}\right)=\frac{k !}{i_{1} ! \cdots i_{\ell-1} !\left(k-i_{1}-\cdots-i_{\ell-1}\right) !} .
$$

The first equation has a solution $b_{0}(y)=y^{\frac{n}{k}}$. Then, as val $a_{1}(y) \geqslant n-\frac{n}{k}$, we have $b_{1}(y)=\frac{1}{k} \frac{a_{1}(y)}{b_{0}(y)^{k-1}} \in F[y]$ ( $k$ is invertible in $F$ ). Next we compute $b_{2}(y), \ldots$ by induction using the fact that system $(\underline{\mathcal{S}})$ is triangular. Suppose that $b_{0}(y), b_{1}(y), \ldots, b_{\ell-1}(y)$ have been found. System $(\underline{\mathcal{S}})$ provides the relation

$$
a_{\ell} \bumpeq k b_{0}^{k-1} b_{\ell}+\sum c_{i_{1} \ldots i_{\ell-1}} b_{0}^{i_{0}} b_{1}^{i_{1}} \cdots b_{\ell-1}^{i_{\ell-1}}
$$

Since $b_{0}(y)=y^{\frac{n}{k}}$, this means that the polynomials $k y^{n-\frac{n}{k}} b_{\ell}(y)$ and $a_{\ell}-$ $\sum c_{i_{1} \ldots i_{\ell-1}} b_{0}^{i_{0}} b_{1}^{i_{1}} \cdots b_{\ell-1}^{i_{\ell-1}}$ have equal coefficients associated to monomials $y^{i}$ with $i \geqslant n-\frac{n}{k}$, whence $b_{\ell}(y)$ is uniquely determined. We have proved that system (S) has a unique solution $\left(b_{0}(y), b_{1}(y), \ldots, b_{\frac{m}{k}}(y)\right)$. 
Finally, we need to prove that $\operatorname{deg} b_{i} \leqslant \frac{n}{k}+i$ for $0 \leqslant i \leqslant \frac{m}{k}$. We have $b_{0}(y)=y^{\frac{n}{k}}$, so that $\operatorname{deg} b_{0}=\frac{n}{k}$ and $b_{1}(y)=\frac{1}{k} \frac{a_{1}(y)}{\left(y^{\frac{n}{k}}\right)^{k-1}} ;$ thus, $\operatorname{deg} b_{1} \leqslant \operatorname{deg} a_{1}-n+\frac{n}{k} \leqslant$ $n+1-n+\frac{n}{k}=\frac{n}{k}+1$. Then, by induction we get

$$
\begin{aligned}
\operatorname{deg} b_{0}^{i_{0}} b_{1}^{i_{1}} \cdots b_{\ell-1}^{i_{\ell-1}} & \leqslant i_{0}\left(\frac{n}{k}+0\right)+i_{1}\left(\frac{n}{k}+1\right)+\cdots+i_{\ell}\left(\frac{n}{k}+\ell\right) \\
& =\frac{n}{k}\left(i_{0}+i_{1}+\cdots+i_{\ell}\right)+i_{1}+2 i_{2}+\cdots+(\ell-1) i_{\ell-1} \\
& =\frac{n}{k} k+\ell \\
& =n+\ell .
\end{aligned}
$$

We also find $\operatorname{deg} a_{\ell} \leqslant n+\ell$ so that $\operatorname{deg} b_{\ell} \leqslant \frac{n}{k}+\ell$.

\section{Strict SUM OF $k$ TH POWERS}

This section is devoted to the proof of the main theorem:

Theorem 8. Let $F$ be a field with more than $k$ elements, whose characteristic does not divide $k$, such that each element of $F$ can be written as a sum of $w_{F}(k) k$ th powers of elements of $F$. Each polynomial $P(x, y) \in F[x, y]$ of degree $d \geqslant 2 k^{4}$ is the sum of $k$ th powers

$$
P(x, y)=Q_{1}(x, y)^{k}+\cdots+Q_{s}(x, y)^{k},
$$

of polynomials $Q_{i} \in F[x, y]$ with $\operatorname{deg} Q_{i}^{k} \leqslant d+k^{3}$ and $s \leqslant 2 k^{3} \ln \left(\frac{d}{k}+1\right) \ln (2 k)+$ $7 k^{4} \ln (k) w_{F}(k)^{2}$.

The bound for $s$ is derived from a sharper bound given at the end of the proof. We start by sketching the proof by pictures; see Figure 5.
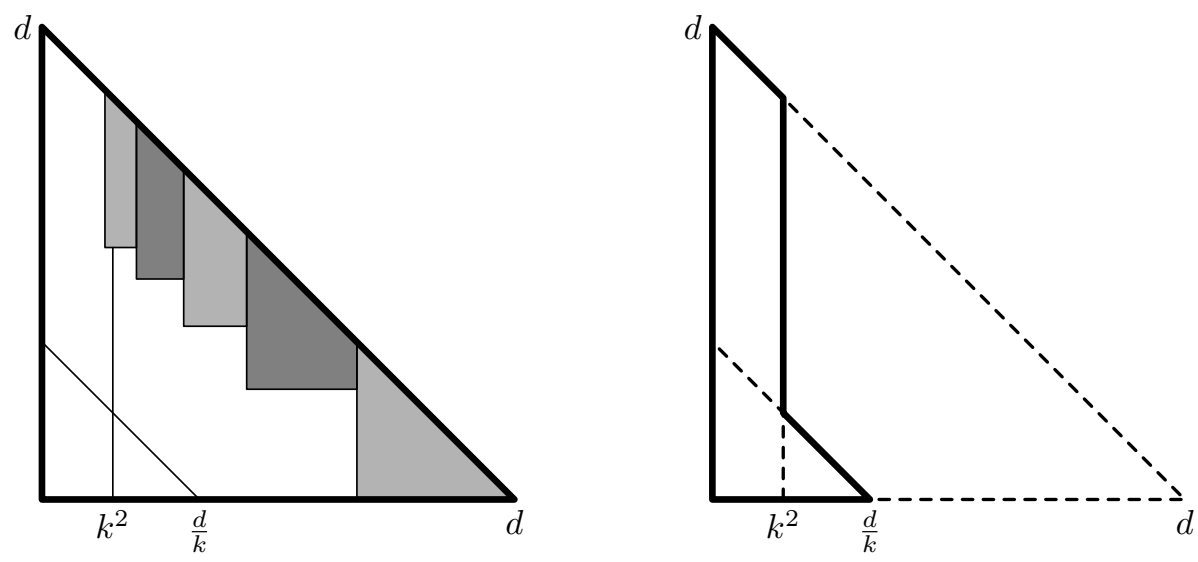

FIGURE 5

We consider the Newton polygon of $P$; it is included in a large triangle (see the left-hand figure in Figure 5). We first cut off trapeziums corresponding to monomials of higher degree. Each trapezium corresponds to a polynomial $Q_{i}^{k}$ computed by an approximate $k$ th root as explained in Section 4 This enables us to lower the 
degree of $P$, except for monomials whose degree in $x$ is less than $k^{2}$, which will be treated at the end. We iterate this process until we get a polynomial of degree less than $\frac{d}{k}$ (right-hand figure in Figure 5) to which we will apply Corollary 4 ,

Notation. We will denote by $\lceil x\rceil_{k}=k\left\lceil\frac{x}{k}\right\rceil$ the least integer greater than or equal to $x$ and divisible by $k$.

First step: lower the degree. Set $d=\operatorname{deg} P, m_{0}=\lceil d\rceil_{k}$ and $P_{0}:=P$. We apply Proposition 7 to $P_{0}=P$, with $P_{0}$ considered as a polynomial of total degree $\leqslant m_{0}$ and $m=m_{0}, n=0$. This yields a polynomial $Q_{0}(x, y)$ such that $\operatorname{deg}_{x}(P+$ $\left.x^{m_{0}}-Q_{0}^{k}\right)<m_{0}-\frac{m_{0}}{k}$. That is to say, we have canceled a trapezium, which is the triangle $\left(m_{0}, 0\right),\left(m_{0}-\frac{m_{0}}{k}, 0\right),\left(m_{0}-\frac{m_{0}}{k}, \frac{m_{0}}{k}\right)$.

We then set $m_{1}=\left\lceil m_{0}\right\rceil_{k}-\frac{\left\lceil m_{0}\right\rceil_{k}}{k}$ and $P_{1}=P_{0}+x^{m_{0}}-Q_{0}^{k}$. Noting that $\operatorname{deg}_{x} P_{1}<m_{1}$, we apply Proposition 7 to $P_{1}$.

To iterate the process, consider the decomposition $P_{i}=P_{i}^{\prime}+x^{m_{i}} \cdot P_{i}^{\prime \prime}$ with $\operatorname{deg}_{x} P_{i}^{\prime}<m_{i}$. We apply Proposition 7 to $P_{i}^{\prime}$ (with $m=\left\lceil m_{i}\right\rceil_{k}$ and $n=n_{i}$ such that $\left\lceil m_{i}\right\rceil_{k}+n_{i}=m_{0}$ ), which yields $Q_{i}$ such that $P_{i}^{\prime}+x^{\left\lceil m_{i}\right\rceil_{k}} y^{n_{i}}-Q_{i}^{k}$ has no monomials in the corresponding trapezium whose $x$-coordinates are in between $\left\lceil m_{i}\right\rceil_{k}$ and $m_{i+1}:=\left\lceil m_{i}\right\rceil_{k}-\frac{\left\lceil m_{i}\right\rceil_{k}}{k}$. Notice that $P_{i+1}:=P_{i}^{\prime}+x^{\left\lceil m_{i}\right\rceil_{k}} y^{n_{i}}-Q_{i}^{k}+x^{m_{i}} \cdot P_{i}^{\prime \prime}$ also does not have monomials in this trapezium.

Here is an example; see Figure 6. Set $d=45$ and $k=3$. Then we get $m_{0}=45$, $m_{1}=30, m_{2}=20, m_{3}=14, m_{4}=10, m_{5}=8$, and then we stop since $m_{5}<k^{2}$. This implies that the first trapezium has its $x$-coordinates in between 45 and 30 , the second one between 30 and $20, \ldots$. The height of the left side of each trapezium is always $\frac{d}{k}=15$.

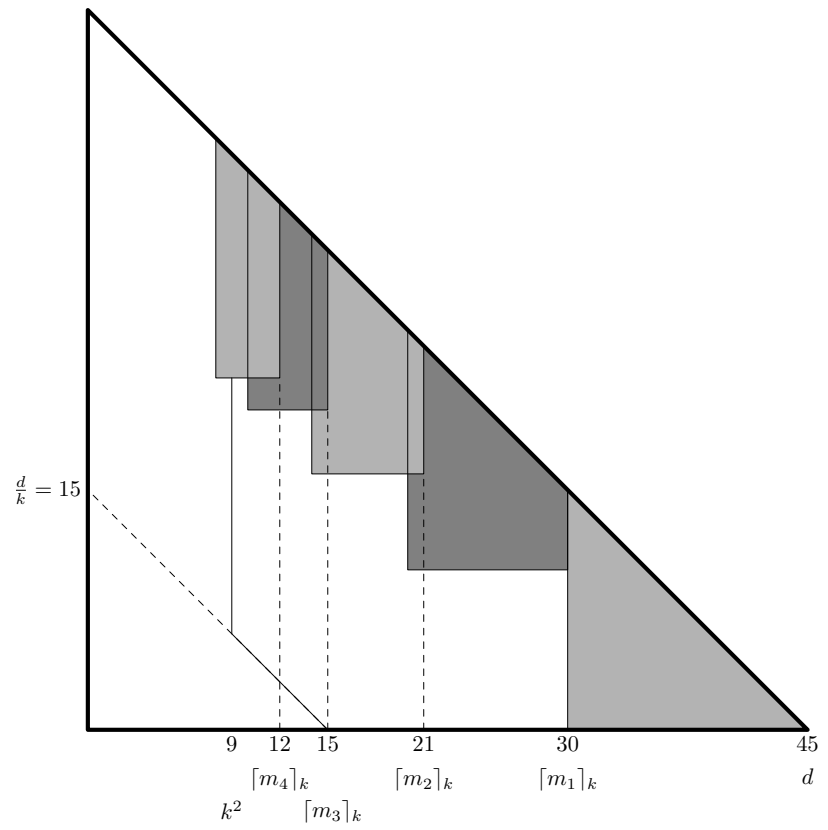

Figure 6 
End of iterations. We iterate the process until we reach monomials whose degree in $x$ is less than $k^{2}$. That is to say, we look for $\ell$ such that $m_{\ell} \leqslant k^{2}$.

First notice that

$$
\begin{aligned}
m_{i+1} & =\left\lceil m_{i}\right\rceil_{k}-\frac{\left\lceil m_{i}\right\rceil_{k}}{k} \\
& =(k-1)\left\lceil\frac{m_{i}}{k}\right\rceil \\
& \leqslant\left(1-\frac{1}{k}\right) m_{i}+k-1 .
\end{aligned}
$$

Then, by induction,

$$
\begin{aligned}
m_{i} & \leqslant\left(1-\frac{1}{k}\right)^{i} m_{0}+(k-1)\left(1+\left(1-\frac{1}{k}\right)+\left(1-\frac{1}{k}\right)^{2}+\cdots+\left(1-\frac{1}{k}\right)^{i-1}\right) \\
& \leqslant\left(1-\frac{1}{k}\right)^{i} m_{0}+k(k-1) \\
& \leqslant(d+k) e^{-\frac{i}{k}}+k(k-1), \quad \text { since }\left(1-\frac{1}{k}\right) \leqslant e^{-\frac{1}{k}} .
\end{aligned}
$$

Now, for $\ell \geqslant k \ln \left(\frac{d}{k}+1\right)$ we get $m_{\ell} \leqslant k^{2}$.

Fall of the total degree. At the end of the first series of iterations the total degree (of the monomials whose degree in $x$ is greater than or equal to $k^{2}$ ) falls (see Figure (7).

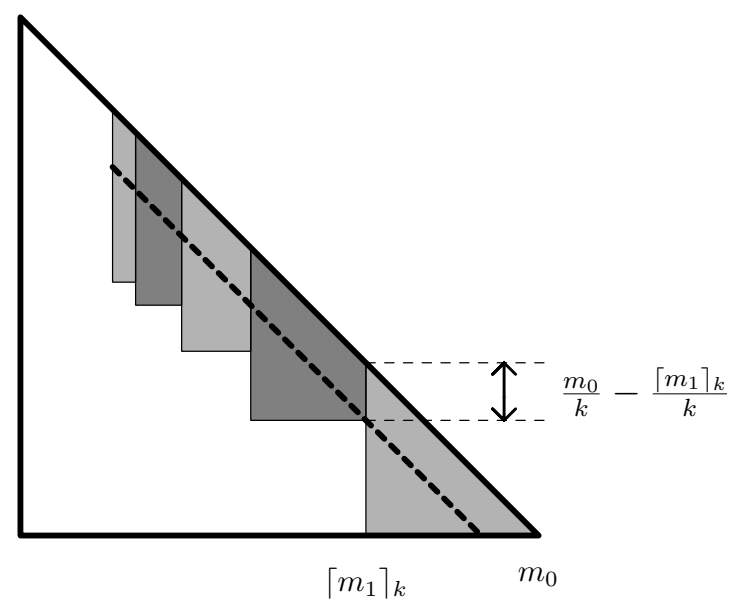

Figure 7 
We give a lower bound for this fall $\delta_{0}$ of the degree (starting from degree $m_{0}$ ):

$$
\begin{aligned}
\delta_{0} & \geqslant \frac{m_{0}}{k}-\frac{\left\lceil m_{1}\right\rceil_{k}}{k} \\
& =\left\lceil\frac{d}{k}\right\rceil-\left\lceil\frac{k\left\lceil\frac{d}{k}\right\rceil-\left\lceil\frac{d}{k}\right\rceil}{k}\right\rceil \quad\left(\text { since } d=\left\lceil m_{0}\right\rceil_{k}\right) \\
& \geqslant\left\lfloor\frac{\left\lceil\frac{d}{k}\right\rceil}{k}\right\rfloor \\
& \geqslant \frac{d}{k^{2}}-1 .
\end{aligned}
$$

Therefore the total degree, starting now from degree $d$, of the monomials whose degree in $x$ is more than $k^{2}$ has fallen by more than $\delta \geqslant \frac{d}{k^{2}}-k$.

Iteration of the fall. Set $d_{0}=d$. At each series of iterations the degree (of the monomials whose degree in $x$ is greater than or equal to $k^{2}$ ) falls from $d_{i}$ to $d_{i+1}:=d_{i}-\left\lfloor\frac{d_{i}}{k^{2}}-k\right\rfloor \leqslant\left(1-\frac{1}{k^{2}}\right) d_{i}+k$, so that (by a computation similar to the one for $m_{i}$ above) $d_{i} \leqslant d e^{-\frac{i}{k^{2}}}+k^{3}$. Suppose that $d \geqslant 2 k^{4}$, so that $\frac{d}{2 k}+k^{3} \leqslant \frac{d}{k}$. Then for $\ell \geqslant k^{2} \ln (2 k)$, we get $d_{\ell} \leqslant \frac{d}{k}$. Each fall of the degree needs less than $k \ln \left(\frac{d}{k}+1\right)$ iterations, so that we need to apply Proposition 7 many times to get a total of $s_{0}=2 k \ln \left(\frac{d}{k}+1\right) \times k^{2} \ln (2 k) k$ th powers.

Monomials of low degree in $x$. At this point, we have written $P=\sum_{i=1}^{s_{0}} Q_{i}^{k}+$ $P_{1}+P_{2}$, where $Q_{1}, \ldots, Q_{s_{0}}, P_{1}, P_{2} \in F[x, y]$ are such that $\operatorname{deg} Q_{i}^{k} \leqslant\lceil d\rceil_{k}, \operatorname{deg}_{x} P_{1}<$ $k^{2}, \operatorname{deg} P_{2} \leqslant \frac{d}{k}$ (see the right-hand picture in Figure [5). By Corollary 4 we can write $P_{2}$ as a sum $P_{2}=\sum_{i=1}^{s_{2}} Q_{i, 2}^{k}$ of $s_{2} \leqslant k w_{F}(k)$ terms and $\operatorname{deg} Q_{i, 2}^{k} \leqslant k\left\lceil\frac{d}{k}\right\rceil=$ $\lceil d\rceil_{k}<d+k$.

Now write $P_{1}(x, y)=\sum_{0 \leqslant j<k^{2}} x^{j} R_{j}(y)$, where $R_{j} \in F[y]$ with $\operatorname{deg} R_{j} \leqslant d-j$. By Corollary 4, write each $x^{j}$ as the sum of $k w_{F}(k)$ terms of degree $\leqslant j k$. Then, for each $R_{j}(y)$ we apply the result in one variable [7, Theorem 1.4 (iii)] (or we can do similar work as before) so that we can write (since $\left.d \geqslant 2 k^{4}\right): R_{j}(y)=$ $\sum_{i=1}^{s} S_{i j}^{k}(y)$ with $s \leqslant k\left(w_{F}(k)+3 \ln (k)\right)+2$ and $\operatorname{deg} S_{i j}^{k} \leqslant \operatorname{deg} R_{j}+k-1$. We get $x^{j} R_{j}(y)$ as the sum of $s^{\prime} \leqslant k w_{F}(k)\left(k\left(w_{F}(k)+3 \ln (k)\right)+2\right), k$ th powers of degree $\leqslant j k+\operatorname{deg} R_{j}+k-1 \leqslant d+k^{3}\left(j=0, \ldots, k^{2}-1\right)$. Therefore, $P_{1}=\sum_{i=1}^{s_{1}} Q_{i, 1}^{k}$ with $s_{1} \leqslant k^{3} w_{F}(k)\left(k\left(w_{F}(k)+3 \ln (k)\right)+2\right)$ terms and $\operatorname{deg} Q_{i, 1}^{k} \leqslant d+k^{3}$.

Conclusion. For $d \geqslant 2 k^{4}$ we can write $P(x, y)$ as the sum

$$
P(x, y)=\sum_{i=1}^{s} Q_{i}^{k}(x, y)
$$

such that $\operatorname{deg} Q_{i}^{k} \leqslant d+k^{3}$ and $s \leqslant s_{0}+s_{2}+s_{1}$; that is to say 2

$$
s \leqslant 2 k^{3} \ln \left(\frac{d}{k}+1\right) \ln (2 k)+k w_{F}(k)+k^{3} w_{F}(k)\left(k\left(w_{F}(k)+3 \ln (k)\right)+2\right) .
$$

This yields the announced bound $s \leqslant 2 k^{3} \ln \left(\frac{d}{k}+1\right) \ln (2 k)+7 k^{4} \ln (k) w_{F}(k)^{2}$.

\footnotetext{
${ }^{2}$ This is the bound used to fill the numerical table of the introduction.
} 
Question. Is it possible to have a sum

$$
P(x, y)=\sum_{i=1}^{s} Q_{i}^{k}(x, y)
$$

such that $\operatorname{deg} Q_{i}^{k} \leqslant \operatorname{deg} P+k^{3}$ and a bound $s$ depending only on $k$ and not on $\operatorname{deg} P$ ?

\section{REFERENCES}

[1] M. Bhaskaran, Sums of mth powers in algebraic and abelian number fields. Arch. Math. (Basel) 17 (1966), 497-504; Correction, ibid. 22 (1971), 370-371. MR0204400 (34:4242). MR0294246(45:3315)

[2] A. Bodin, Decomposition of polynomials and approximate roots. Proc. Amer. Math. Soc. 138 (2010), 1989-1994. MR2596034 (2011b:13024)

[3] M. Car, New bounds on some parameters in the Waring problem for polynomials over a finite field. Contemporary Mathematics 461, Amer. Math. Soc., 2008, 59-77. MR2436325 (2009j:11190)

[4] M. Car, L. Gallardo, Sums of cubes of polynomials. Acta Arith. 112 (2004), 41-50. MR2040591(2005a:11190)

[5] G. Effinger, D. Hayes, Additive number theory of polynomials over a finite field. Oxford Mathematical Monographs, Clarendon Press, Oxford (1991). MR,1143282 (92k:11103)

[6] L. H. Gallardo, On the restricted Waring problem over $\mathbf{F}_{2^{n}}[t]$. Acta Arith. 92 (2000), 109-113. MR.1750311 (2001e:11123)

[7] L. Gallardo, L. Vaserstein, The strict Waring problem for polynomial rings. J. Number Theory 128 (2008), 2963-2972. MR2464848(2009j:11191)

[8] R.M. Kubota, Waring's problem for $\mathbf{F}_{q}[x]$. Dissertationes Math. (Rozprawy Mat.) 117 (1974). MR0376581 (51:12756)

[9] Y.-R. Liu, T. Wooley, The unrestricted variant of Waring's problem in function fields. Funct. Approx. Comment. Math. 37 (2007), 285-291. MR2363827 (2009d:11139)

[10] R.E.A.C Paley, Theorems on polynomials in a Galois field. Quarterly J. of Math. 4 (1933), $52-63$.

[11] L.N. Vaserstein, Waring's problem for algebras over fields. J. Number Theory 26 (1987), 286-298. MR.901241 (89e:11059)

[12] L.N. Vaserstein, Sums of cubes in polynomial rings. Math. Comput. 56 (1991), 349-357. MR $1052104(91 \mathrm{e}: 11106)$

[13] L.N. Vaserstein, Ramsey's theorem and Waring's problem. In The Arithmetic of Function Fields (eds. D. Goss et al.), de Gruyter, New York-Berlin, 1992. MR1196531 (94a:11155)

[14] W.A. Webb, Waring's problem in $G F[q, x]$. Acta Arith. 22 (1973), 207-220. MR0313190 $(47: 1745)$

Laboratoire Paul Painlevé, UfR Mathématiques, Université Lille 1, 59655 Villeneuve D'Ascq Cedex, France

E-mail address: Arnaud.Bodin@math.univ-lille1.fr

Université Paul Cézanne, Faculté de Saint-Jérôme, 13397 Marseille Cedex, France

E-mail address: Mireille.Car@univ-cezanne.fr 